\section{Valoración de un programa para la provisión de sillas de ruedas adecuadas para pacientes con lesión medular tomando como indicadores la calidad de vida y la prevención de úlceras por presión}

\author{
Assessment of Wheelchair Provision Program to Spinal \\ Cord Injuries Wheelchair Users, applying Quality of \\ Life and Pressure Ulcer Prevention as Indicators \\ (D) Adriana Lorena Fajardo Martos ${ }^{1}$, Diana Alicia Gayol Mérida ${ }^{2}$, \\ (D) Hugo Alexer Pérez Vicente ${ }^{3}$, Ana Moreno Hernández ${ }^{4}$, Ramiro Pérez Zavala ${ }^{5}$
}

\section{RESUMEN}

Introducción. La lesión medular afecta la calidad de vida y el estado de salud de la persona que la padece. Por su parte, el uso de una silla de ruedas adecuada a las necesidades y características particulares de cada individuo puede aumentar los cuidados en la salud, prevenir complicaciones como las úlceras por presión e incrementar la calidad de vida.

Objetivo. Valorar la percepción de la calidad de vida y dar seguimiento a la formación de úlceras por presión como indicadores del impacto que tiene un programa basado en las "Pautas de suministro de sillas de ruedas manuales en entornos de menores recursos" de la OMS.

Material y métodos. A un grupo piloto de lesionados medulares se les otorgó una silla de ruedas adecuada; se les aplicaron los cuestionarios CHART-SF y WHOQOL-BREF para medir su calidad de vida y se les hizo seguimiento del desarrollo de úlceras por presión durante 12 meses. Resultados. Los principales hallazgos fueron una mejor percepción de la movilidad en el CHART-SF, mientras que en el WHOQOL-BREF el grupo no percibió ningún cambio, ni positivo ni negativo, en su calidad de vida. Los pacientes no desarrollaron úlceras por presión durante el estudio.

Conclusiones. Los cuestionarios aplicados no son suficientemente sensibles para medir la calidad de vida en pacientes con lesión medular.

Palabras clave. Silla de ruedas, calidad de vida, úlcera por presión.

\section{ABSTRACT}

Introduction. Spinal cord injury affects the quality of life and health of a person. An appropriate wheelchair can increase health care, prevent complications like pressure ulcers and improve the quality of life.

Objective. To assessment quality of life perception and developing of pressure ulcer as indicators of a wheelchair supplying program to spinal cord injuries based of WHO program.

Material and methods. An appropriate wheelchair was given to a pilot group with spinal cord injury, thus the quality of life was measured by CHART-SF and WHOQOL-BREF questionnaires. A 12-month follow-up was done to detect the development of pressure ulcers. Results. CHART-SF questionnaire outcomes show that the group perceived a greater mobility, while in the WHOQOL-BREF questionnaire, the group did not perceive any change, whether positive or negative, with regards to quality of life. The group did not develop pressure ulcers. Conclusions. The questionnaires outcomes are not sensitive enough to measure quality of life changes in patients of spinal cord injury.

Keywords. Wheelchairs; quality of life; pressure ulcer.
Autores:

Ingeniera Biomédica, Maestra en Ingeniería de Calidad. Departamento de Ingeniería Química, Industrial y de Alimentos, Universidad Iberoamericana, Ciudad de México, México.

2Ingeniera Biomédica, Maestra en Ingeniería de Calidad. Sistema Nacional para el Desarrollo Integral de la Familia. Departamento de Enseñanza e Investigación, Centro Nacional Modelo de Atención, Investigación y Capacitación para la Rehabilitación e Integración Educativa "Gaby Brimmer", Ciudad de México, México.

3Ingeniero Industrial, Maestro en Ciencias en Ingeniería de Sistemas, Doctor en Ingeniería Industrial. Departamento de Ingeniería Química, Industrial y de Alimentos, Universidad Iberoamericana, Ciudad de México, México.

4Ingeniera Biomédica, Maestra en Ciencias en Cinemática Humana. Laboratorio de Análisis de Movimiento Humano, Dirección de Investigación, Instituto Nacional de Rehabilitación Luis Guillermo Ibarra Ibarra, Ciudad de México, México.

${ }^{5}$ Médico Cirujano, Especialista en Medicina de Rehabilitación, Sub especialista en Rehabilitación Neurológica. Servicio de Lesión Medular, División de Rehabilitación Neurológica, Instituto Nacional de Rehabilitación Luis Guillermo Ibarra Ibarra, Ciudad de México, México.

\section{Correspondencia:}

Diana Alicia Gayol Mérida. Sistema Nacional para el Desarrollo Integral de la Familia. Departamento de Enseñanza e Investigación, Centro Nacional Modelo de Atención, Investigación y Capacitación para la Rehabilitación e Integración Educativa "Gaby Brimmer", Eje 7-Sur Zapata, Col. Santa Cruz Atoyac, Alcaldía Benito Juárez, CP 03310, Ciudad de México, México. Correo electrónico:

diana.gayol@outlook.com

Recibido: 25.11 .21

Aceptado:21.01.21

\section{Citación:}

Fajardo Martos AL, Gayol Mérida DA, Pérez Vicente HA, Moreno Hernández A, Pérez Zavala R. Valoración de un programa para la provisión de sillas de ruedas adecuadas para pacientes con lesión medular tomando como indicadores la calidad de vida y la prevención de úlceras por presión. Rev Col Med Fis Rehab 2021;31(2): 136-145.

https://doi.org/10.28957/rcmfr.v31n2a4

Conflictos de interés:

Ninguno declarado por los autores. 


\section{INTRODUCCIÓN}

Según la Organización Panamericana de la Salud (OPS) ${ }^{1}$, se estima que casi el $12 \%$ de la población de América Latina y el Caribe vive con al menos una discapacidad, lo que representa alrededor de 66 millones de personas; en el mismo documento, esta entidad también indica que una de las discapacidades más frecuentes es la motriz ${ }^{1}$, siendo la lesión medular la más común en personas adultas en edad productiva (18-32 años) y de sexo masculino ${ }^{2}$.

La lesión medular es un evento catastrófico, disruptivo e inesperado en la vida de una persona y por tanto puede ser devastador en términos económicos y sociales tanto para el paciente como para su familia, lo cual, a su vez, afecta la calidad de vida, no solo de quien sufrió la lesión medular sino también de sus familiares, y su entorno social.

La calidad de atención en el cuidado de los pacientes con lesión medular se refleja en las estadísticas de supervivencia posterior a la lesión. De esta forma, el retraso en la atención especializada puede ocasionar que los pacientes con este tipo de lesión desarrollen complicaciones como úlceras por presión, osificación heterotópica, complicaciones urinarias, entre otras, lo cual puede generar una larga estancia hospitalaria ${ }^{2}$. En consecuencia, es prioridad diseñar estrategias que prevengan estas complicaciones, ya que por ejemplo las úlceras por presión las desarrollan entre el 31\% y el $79 \%$ de los pacientes con lesión medular ${ }^{3,4}$.

En un estudio realizado por Zakrasek et al. ${ }^{5}$ en varios países como Vietnam, Chile e India, se encontró que en las personas con alguna discapacidad motriz que recibieron una silla de ruedas adecuada a sus necesidades disminuyó la incidencia de úlceras por presión. Este estudio además sugirió que la disminución puede deberse al incremento en la movilidad con la silla de ruedas y en el acceso a los cuidados de la salud, e identificó un decremento en el dolor, una actitud más positiva hacia la vida y, como consecuencia, una mejora en la calidad de vida.
En relación con las sillas ruedas apropiadas a las necesidades de cada usuario, la Organización Mundial de la Salud (OMS) desarrolló una guía estandarizada llamada "Pautas para el suministro de sillas de ruedas manuales en entornos de menores recursos" 6 , la cual va acorde con las Normas Uniformes de las Naciones Unidas sobre la Igualdad de Oportunidades para personas con discapacidad, la Convención de los Derechos de las Personas con discapacidad y la Resolución WHA58.23 de la Asamblea Mundial de la Salud ${ }^{7,8}$. En dicha guía se establecen ocho pasos para seleccionar la silla de ruedas indicada para un paciente: derivación y cita; estudio; prescripción; financiamiento y pedido; preparación del producto; prueba de ajuste; capacitación del usuario, y seguimiento, mantenimiento y reparaciones ${ }^{6}$.

Por otro lado, la calidad de vida es definida por la OMS en función de cómo el individuo percibe el lugar que ocupa en su entorno cultural y en el sistema de los valores en que vive, así como en relación con sus objetivos, expectativas, criterios y preocupaciones, en conjunto con la salud física, el estado psicológico, el grado de independencia, las relaciones sociales, entre otras $^{8}$.

Cualquier intervención relacionada con la prevención o el tratamiento de una enfermedad o discapacidad tiene como meta preservar la calidad de vida del paciente, por lo que se requieren evaluaciones sobre la mejora o el deterioro de este aspecto y del estado funcional de cada individuo. En la investigación de Velarde-Jurado $\&$ Ávila-Figueroa ${ }^{9}$ se menciona que para medir adecuadamente la calidad de vida de un paciente es necesario emplear cuestionarios que ayuden a cuantificar los problemas de salud.

En este sentido, el objetivo del presente estudio fue valorar la percepción de la calidad de vida y dar seguimiento a la formación de úlceras por presión como indicadores del impacto que tiene un programa basado en las "Pautas de suministro de sillas de ruedas manuales en entornos de menores recursos" de la OMS para la provisión de sillas de ruedas a un

Valoración de un programa para la provisión de sillas de ruedas adecuadas para pacientes con lesión medular tomando como indicadores la calidad de vida y la prevención de úlceras por presión 
grupo piloto de pacientes con lesión medular según sus necesidades y características particulares, esto teniendo en cuenta que una silla de ruedas adecuada puede mejorar la calidad de vida de los pacientes con lesión medular al brindarles una mayor independencia y movilidad $^{10}$. Además, una silla de ruedas que no esté diseñada o no sea adecuada a las necesidades del paciente puede asociarse a la formación de úlceras por presión ${ }^{11}$.

\section{MATERIALES Y MÉTODOS}

Estudio descriptivo longitudinal realizado en el Instituto Nacional de Rehabilitación Luis Guillermo Ibarra Ibarra (INR-LGII), México, en el año 2017. Se reclutaron a 12 pacientes con lesión medular evaluados por médicos especialistas en rehabilitación para que participaran de forma voluntaria en la investigación y firmaran un consentimiento informado. El tamaño de la muestra se estableció por conveniencia a partir del monto asignado para la compra de sillas de ruedas. El estudio cumplió con los criterios de investigación en seres humanos del INR-LGII.

Los criterios de inclusión fueron: 1) que el paciente hubiera sufrido la lesión medular entre un mes y año y medio antes de la realización del estudio, 2) que la lesión medular aguda se ubicara a nivel torácico bajo o lumbar y 3) que el voluntario tuviera control del tronco y manipulara su silla de ruedas de forma independiente.

A los participantes, que eran atendidos en el Programa Intensivo de Rehabilitación para Pacientes con Lesión Medular otorgado por el Servicio de Lesión Medular del INR-LGII, se les otorgó una silla de ruedas ajustada a sus necesidades y características particulares de acuerdo al programa de los ocho pasos de la OMS y se les aplicaron los cuestionarios CHART-SF (Craig Handicap Assessment and Reporting Technique Short Form) y WHOQOLBREF (World Health Organization Quality of Life), en su versión en español, para medir la percepción de la calidad de vida con respecto a la silla de ruedas entregada.
Asimismo, se hizo seguimiento por parte del Servicio de Lesión Medular sobre el estado general de salud de los pacientes, así como un monitoreo para detectar la formación de úlceras por presión. Los periodos de seguimiento y aplicación de cuestionarios luego de la entrega de la silla de ruedas fueron a los 3, 6, 9 y 12 meses de haber iniciado el estudio.

El cuestionario CHART-SF evalúa las dimensiones de la discapacidad propuestas por la OMS: independencia física, movilidad, independencia cognitiva, ocupación, integración social y autosuficiencia económica ${ }^{12}$, mientras que el cuestionario WHOQOL-BREF, desarrollado por la OMS, evalúa los dominios de la salud física y psicológica, las relaciones sociales y el medio ambiente ${ }^{13}$.

Los datos recopilados con ambos cuestionarios fueron transformados para su análisis en una escala medible; es decir, dado que el CHART-SF usa una escala del 0 a 100 para cada dimensión y el WHOQOL -BREF tiene dos escalas, de este último se escogió la que va de 0 a 100 para cada dominio y que así los resultados fueran equiparables. En el cuestionario CHART-SF se hizo un ajuste al excluir la dimensión autosuficiencia económica debido a que la transformación de esta incluye el índice de pobreza basado en el número de integrantes de una familia (así se calcula en Estados Unidos) y en México la construcción de este indicador considera otros factores.

El análisis de los dos cuestionarios consistió en un análisis descriptivo de cada dimensión o dominio (según sea el caso) en cada periodo estudiado. Se tuvieron dos tipos de comparaciones: a) absoluta, que consiste en comparar todos los periodos de seguimiento a partir del mes 3 con el periodo basal (cuando se entregó la silla de ruedas al inicio del estudio), y b) relativa, que consiste en comparar las diferencias entre un periodo y otro, por ejemplo, basal vs. mes 3 , mes 3 vs. mes 6 , y así sucesivamente hasta completar las comparaciones al mes 12. La prueba de rangos con signos de Wilcoxon se aplicó para probar la hipótesis estadística para grupos pareados y posteriormente 
se aplicaron las pruebas Shapiro-Wilks para revisar distribución normal y de Bartlett para verificar igualdad de varianzas en los datos.

El análisis estadístico se realizó en el software R (software de ambiente libre especializado en estadística computacional) y se corroboró con el software SPSS.

\section{RESULTADOS}

En la Tabla 1 se muestran las características sociodemográficas y antropométricas de los participantes. Se excluyó un voluntario del estudio ya que no cumplió con las citas de seguimiento.

Durante el seguimiento se observó que ningún participante del estudio desarrolló úlceras por presión.

Para el cuestionario CHART-SF se evaluaron los supuestos de normalidad e igualdad de varianzas. En la Tabla 2 se observan las dimensiones que rechazan la hipótesis nula en algunos periodos con un nivel de significancia de 0,05: Independencia física en el mes 3, Independencia cognitiva en el periodo basal o inicio del estudio, Ocupación en el periodo basal o inicio del estudio y en los meses 3, 9 y 12 e Integración social en todos los periodos. Por consiguiente, se puede decir que la mayoría de los datos no cumplen con el supuesto de normalidad.
Respecto al supuesto de igualdad de varianzas, en el cuestionario CHART-SF se analizaron las dos comparaciones de periodos: en el comparativo absoluto (Tabla 3) ninguna de las dimensiones y periodos comparados rechazaron la hipótesis nula con un nivel de significancia del 0,05, excepto la dimensión de Integración social en el periodo basal a mes 3 que sí rechazó la hipótesis nula $(p=0,003)$, y en el comparativo relativo (Tabla 4) solamente la dimensión Integración social para el periodo basal a mes 3 rechazó la hipótesis nula ( $p=0,003)$. Para el resto de las dimensiones, con sus respectivos periodos comparativos, no se rechazó la hipótesis nula. Se puede decir entonces que los resultados del cuestionario cumplen con este supuesto, aunque estadísticamente se sabe que no es un supuesto determinante.

Debido a que no se cumplió con el supuesto de normalidad, se utilizó la prueba de Wilcoxon para el análisis de los datos del cuestionario CHART-SF. En la Tabla 5 se aprecia que, para la comparación absoluta, la hipótesis nula no se rechazó con un nivel de significancia del 0,05 en todos los casos, lo que significa que no hay evidencia estadística que demuestre algún cambio entre la aplicación de los cuestionarios; lo mismo sucede para la comparación relativa (Tabla 6), donde no se rechazó la hipótesis nula para todas las dimensiones y periodos comparados, a excepción de la dimensión Movilidad en el periodo del mes 9 al mes 12, que sí la rechaza $(p=0,027)$.

Tabla 1. Características sociodemográficas y antropométricas de los participantes.

\begin{tabular}{|l|l|c|}
\hline \multicolumn{2}{|l|}{ Total de voluntarios } & \multicolumn{1}{|c|}{11} \\
\hline Sexo & Masculino \\
& femenino & 10 \\
\hline Edad (años) & \multicolumn{1}{|l}{ Torácico alto } & $31.9 \pm 7.7$ \\
\hline \multirow{2}{*}{ Nivel de lesión medular } & Torácico bajo & 6 \\
& Lumbar & 2 \\
\hline Comorbilidades & Hipertensión & 1 \\
\hline Tiempo de evolución de la lesión medular (años) & 0 \\
\hline
\end{tabular}

Fuente: elaboración propia. 
Tabla 2. Dimensiones que rechazan la hipótesis nula del supuesto de normalidad del cuestionario CHART-SF.

\begin{tabular}{|l|c|c|c|c|c|}
\hline \multicolumn{1}{|c|}{ Dimensión } & Basal & Mes 3 & Mes 6 & Mes 9 & Mes 12 \\
\hline Independencia física & 0.282 & 0.001 & 0.151 & 0.166 & 0.121 \\
\hline Movilidad & 0.495 & 0.317 & 0.799 & 0.702 & 0.541 \\
\hline Independencia cognitiva & 0.033 & 0.799 & 0.900 & 0.221 & 0.870 \\
\hline Ocupación & 0.024 & 0.048 & 0.105 & 0.006 & 0.001 \\
\hline Integración social & 0.000 & 0.000 & 0.000 & 0.003 & 0.000 \\
\hline
\end{tabular}

Fuente: elaboración propia.

Tabla 3. Prueba de igualdad de varianzas (absoluto) para CHART-SF.

\begin{tabular}{|l|c|c|c|c|}
\hline Dimensión & Basal a mes 3 & Basal a mes 6 & Basal a mes 9 & Basal a mes 12 \\
\hline Independencia física & 0.328 & 0.139 & 0.869 & 0.629 \\
\hline Movilidad & 0.649 & 0.656 & 0.565 & 0.243 \\
\hline Independencia cognitiva & 0.092 & 0.073 & 0.124 & 0.097 \\
\hline Ocupación & 0.714 & 0.306 & 0.877 & 0.651 \\
\hline Integración social & 0.003 & 0.142 & 0.346 & 0.246 \\
\hline
\end{tabular}

Fuente: elaboración propia.

Tabla 4. Prueba de igualdad de varianzas (relativo) para CHART-SF.

\begin{tabular}{|l|c|c|c|c|}
\hline Dimensión & Basal a mes 3 & Mes 3 a mes 6 & Mes 6 a mes 9 & Mes 9 a mes 12 \\
\hline Independencia física & 0.328 & 0.628 & 0.223 & 0.539 \\
\hline Movilidad & 0.649 & 0.375 & 0.319 & 0.102 \\
\hline Independencia cognitiva & 0.092 & 0.967 & 0.901 & 0.899 \\
\hline Ocupación & 0.714 & 0.175 & 0.413 & 0.775 \\
\hline Integración social & 0.003 & 0.095 & 0.644 & 0.053 \\
\hline
\end{tabular}

Fuente: elaboración propia.

Tabla 5. Prueba Wilcoxon valores p (absoluta) para CHART-SF.

\begin{tabular}{|l|c|c|c|c|}
\hline Dimensión & Basal a mes 3 & Mes 3 a mes 6 & Mes 6 a mes 9 & Mes 9 a mes 12 \\
\hline Independencia física & 0.212 & 0.438 & 0.684 & 0.599 \\
\hline Movilidad & 0.541 & 0.919 & 0.263 & 0.068 \\
\hline Independencia cognitiva & 0.400 & 0.888 & 0.611 & 0.916 \\
\hline Ocupación & 0.678 & 0.286 & 0.917 & 0.080 \\
\hline Integración social & 0.109 & 0.180 & 0.500 & 0.317 \\
\hline
\end{tabular}

Fuente: elaboración propia. 
Tabla 6. Prueba Wilcoxon valores p (relativa) para CHART-SF.

\begin{tabular}{|l|c|c|c|c|}
\hline Dimensión & Basal a mes 3 & Mes 3 a mes 6 & Mes 6 a mes 9 & Mes 9 a mes 12 \\
\hline Independencia física & 0.212 & 0.112 & 0.121 & 0.785 \\
\hline Movilidad & 0.541 & 0.721 & 0.674 & 0.027 \\
\hline Independencia cognitiva & 0.400 & 0.260 & 0.496 & 0.596 \\
\hline Ocupación & 0.678 & 0.063 & 0.799 & 0.144 \\
\hline Integración social & 0.109 & 0.465 & 0.893 & 0.273 \\
\hline
\end{tabular}

Fuente: elaboración propia.

Al igual que para el cuestionario CHARTSF, se evaluaron los supuestos de normalidad y de igualdad de varianzas para las comparaciones absolutas y relativas del cuestionario WHOQOL-BREF. Para el supuesto de normalidad, en la Tabla 7 se muestran los dominios que rechazaron la hipótesis nula en algunos periodos con un nivel de significancia de 0,05, que son: Psicológico en el mes 12 y Medio ambiente en el mes 3. Esto significa que, para los casos mencionados no se cumple con el supuesto de normalidad, es decir, sus datos no provienen de una distribución normal. El resto de los dominios y períodos tienen una distribución semejante a la normal. Por su parte, en el análisis del supuesto de igualdad de varianzas
(Tablas 8 y 9) se observó que la hipótesis nula no se rechazó, con un nivel de significancia del 0,05, por lo tanto se cumple el supuesto de igualdad de varianzas para ambas comparaciones (absoluta y relativa) de todos los dominios.

Finalmente, y debido a que ninguno de los datos se asemeja a una distribución normal, se hizo la prueba Wilcoxon para ambas comparaciones (Tablas 10 y 11) y se estableció que los valores de p resultantes no rechazan la hipótesis nula con un nivel de significancia del 0,05 en los diferentes dominios, lo que sugiere que no hay suficiente evidencia estadística para identificar algún cambio, ya sea positivo o negativo, entre cada periodo de aplicación del cuestionario.

Tabla 7. Dimensiones que rechazan la hipótesis nula del supuesto de normalidad del cuestionario WHOQOL-BREF.

\begin{tabular}{|l|c|c|c|c|c|}
\hline Dimensión & Basal & Mes 3 & Mes 6 & Mes 9 & Mes 12 \\
\hline Salud física & 0.404 & 0.424 & 0.336 & 0.957 & 0.050 \\
\hline Psicológico & 0.230 & 0.742 & 0.554 & 0.911 & 0.013 \\
\hline Relaciones Sociales & 0.741 & 0.543 & 0.314 & 0.131 & 0.674 \\
\hline Medio Ambiente & 0.966 & 0.043 & 0.153 & 0.303 & 0.810 \\
\hline
\end{tabular}

Fuente: elaboración propia.

Tabla 8. Prueba de igualdad de varianzas (absoluto) para WHOQOL-BREF.

\begin{tabular}{|l|c|c|c|c|}
\hline Dimensión & Basal a mes 3 & Basal a mes 6 & Basal a mes 9 & Basal a mes 12 \\
\hline Salud física & 0.984 & 0.734 & 0.787 & 0.822 \\
\hline Psicológico & 0.707 & 0.501 & 0.829 & 0.080 \\
\hline Relaciones sociales & 0.303 & 0.635 & 0.351 & 0.189 \\
\hline Medio ambiente & 0.837 & 0.909 & 0.428 & 0.509 \\
\hline
\end{tabular}

Fuente: elaboración propia. 
Tabla 9. Prueba de igualdad valores p (relativo) para WHOQOL-BREF.

\begin{tabular}{|l|c|c|c|c|}
\hline Dimensión & Basal a mes 3 & Mes 3 a mes 6 & Mes 6 a mes 9 & Mes 9 a mes 12 \\
\hline Salud física & 0.984 & 0.762 & 0.553 & 0.629 \\
\hline Psicológico & 0.707 & 0.784 & 0.653 & 0.138 \\
\hline Relaciones sociales & 0.303 & 0.578 & 0.653 & 0.704 \\
\hline Medio ambiente & 0.837 & 0.757 & 0.505 & 0.161 \\
\hline
\end{tabular}

Fuente: elaboración propia.

Tabla 10. Prueba Wilcoxon valores p (absoluto) para WHOQOL-BREF.

\begin{tabular}{|l|c|c|c|c|}
\hline Dimensión & Basal a mes 3 & Basal a mes 6 & Basal a mes 9 & Basal a mes 12 \\
\hline Salud física & 0.398 & 0.202 & 0.215 & 1.000 \\
\hline Psicológico & 0.677 & 0.959 & 0.758 & 0.092 \\
\hline Relaciones sociales & 0.270 & 0.473 & 0.953 & 0.944 \\
\hline Medio ambiente & 0.779 & 0.282 & 0.823 & 0.859 \\
\hline
\end{tabular}

Fuente: elaboración propia.

Tabla 11. Prueba Wilcoxon valores p (relativo) para WHOQOL-BREF.

\begin{tabular}{|l|c|c|c|c|}
\hline Dimensión & Basal a mes 3 & Mes 3 a mes 6 & Mes 6 a mes 9 & Mes 9 a mes 12 \\
\hline Salud física & 0.398 & 0.347 & 0.682 & 0.106 \\
\hline Psicológico & 0.677 & 0.197 & 0.831 & 0.059 \\
\hline Relaciones Sociales & 0.270 & 0.606 & 0.389 & 0.394 \\
\hline Medio ambiente & 0.779 & 0.098 & 0.039 & 0.734 \\
\hline
\end{tabular}

Fuente: elaboración propia.

\section{DISCUSIÓN}

En este estudio se mostró la aplicación de los ocho pasos para la prescripción de sillas de ruedas propuestos por la OMS en conjunto con el Programa Intensivo de Rehabilitación para Pacientes con Lesión Medular. Asimismo, con la aplicación del cuestionario CHART-SF se encontró una diferencia estadísticamente significativa positiva en la dimensión de Movilidad de la comparación relativa entre el mes 9 y el 12, la cual sugiere que los voluntarios perciben una mayor libertad para movilizarse y para realizar sus actividades de la vida diaria. Este resultado coincide con lo encontrado por Zakrasek et al. ${ }^{5}$ en su estudio. Además, la percepción de una mejora en la dimensión Movilidad podría deberse al entrenamiento intensivo en el uso de la silla de ruedas.

El inconveniente del cuestionario CHARTSF es que todavía no ha sido validado al idioma español; no obstante, se decidió continuar con el estudio considerando al grupo de voluntarios como un grupo piloto con el cual se puede establecer si este puede ser una herramienta viable que detecte de manera oportuna cambios en la calidad de vida en este tipo de pacientes. 
Asimismo, hay que considerar que el presente estudio se hizo bajo una muestra reducida de pacientes a consecuencia de la limitación en el recurso económico asignado para la compra de sillas de ruedas adecuadas a las necesidades y características individuales cada individuo.

Por otro lado, con el cuestionario WHOQOLBREF no se observó ninguna diferencia estadísticamente significativa en los cuatro dominios, ni en las comparaciones absolutas ni en las relativas. Esto se traduce en que los pacientes con lesión medular no perciben diferencia, ya sea positiva o negativa, en su estado de salud, ánimo o autoestima, ni tampoco indican que hayan retomado su vida social o laboral a como era antes de sufrir la lesión medular.

Los resultado obtenidos con los cuestionarios aplicados pueden deberse a que estos son de tipo genérico y a que la lesión medular puede tener un impacto altamente negativo en la calidad de vida y bienestar de las personas que la sufren, aunque no siempre se detecten cambios importantes en la calidad de vida. Por consiguiente, se sugiere el uso o el desarrollo de instrumentos específicos para este tipo de discapacidad.

En relación con el seguimiento que realizaron los médicos especialistas en rehabilitación del estado de salud, se estableció que ningún voluntario tuvo deterioro de su estado de salud y esto se debe al estrecho seguimiento médico que se hizo. Además, ningún paciente desarrolló úlceras por presión, lo que pudo deberse a la combinación de una silla de ruedas adecuada a las necesidades del voluntario y a la intervención mediante el Programa Intensivo de Rehabilitación para Pacientes con Lesión Medular, pues, de acuerdo con Lyder ${ }^{14}$, el no desarrollo de úlceras por presión es un indicativo de la calidad de la atención en los servicios de salud, ya que cada año se destina una cantidad considerable de recursos para su tratamiento.

Una úlcera por presión puede convertirse en una herida crónica que le ocasiona al paciente con lesión medular estrés emocional, malnutrición e infecciones recurrentes que pueden derivar en osteomielitis o incluso la muerte; además, estas lesiones suelen ocasionar exorbitantes implicaciones económicas que afectan tanto al paciente como al sistema de salud que lo atiende ${ }^{15}$, de ahí la importancia de su prevención y tratamiento.

\section{CONCLUSIÓN}

El presente estudio sugiere que la provisión de una silla de ruedas, en conjunto con la implementación de programas de rehabilitación, puede ayudar a la prevención de las úlceras por presión; sin embargo, se debe realizar un seguimiento a largo plazo para conocer si estas medidas ayudan a prevenir otros tipos de padecimientos que afectan a lesionados medulares crónicos y si a largo plazo estos perciben un incremento en su calidad de vida.

\section{FINANCIACIÓN}

El estudio contó con el apoyo económico del Consejo Nacional de Ciencia y Tecnología gracias al proyecto CONACyT-2013-1202273.

\section{RESPONSABILIDADES ÉTICAS}

\section{Protección de personas y animales}

Los autores declaran que para esta investigación no se han realizado experimentos en seres humanos ni en animales.

\section{Confidencialidad de los datos}

Los autores declaran que han seguido los protocolos de su centro de trabajo sobre la publicación de datos de pacientes. 


\section{Derecho a la privacidad y consentimiento informado}

Los autores declaran que en este artículo no aparecen datos que puedan identificar a las personas que respondieron la encuesta; así mismo, se contó con la aprobación de los encuestados para divulgar los datos.

\section{AGRADECIMIENTOS}

Al Consejo Nacional de Ciencia y Tecnología. Los autores dedican este trabajo in memoriam al Dr. Ramiro Pérez Zavala por su compromiso y dedicación para mejorar la calidad de vida de sus pacientes.

\section{CONTRIBUCIÓN DE LOS AUTORES}

Adriana Lorena Fajardo Martos: realizó una contribución sustancial al diseño del artículo, al análisis y a la interpretación de los datos obtenidos del estudio; apoyó la revisión crítica del contenido intelectual del presente trabajo, y participó en la elaboración de la versión final de manuscrito.
Diana Alicia Gayol Mérida: realizó una contribución sustancial al diseño del artículo, al análisis y a la interpretación de los datos obtenidos del estudio; apoyó la revisión crítica del contenido intelectual del presente trabajo, y participó en la elaboración de la versión final de manuscrito.

Hugo Alexer Pérez Vicente: realizó una contribución sustancial al diseño del artículo, al análisis y a la interpretación de los datos obtenidos del estudio; apoyó la revisión crítica del contenido intelectual del presente trabajo, y participó en la elaboración de la versión final de manuscrito.

Ana Moreno Hernández: realizó una contribución sustancial al diseño del estudio clínico y al análisis de los datos obtenidos del estudio; ayudó en la revisión crítica del contenido intelectual del presente trabajo, y participó en la elaboración de la versión final de manuscrito.

Ramiro Pérez Zavala: realizó una contribución sustancial al diseño del estudio clínico y al análisis de los datos obtenidos del estudio, y colaboró con la revisión crítica del contenido intelectual del presente trabajo. 


\section{REFERENCIAS}

1. Organización Panamericana de la Salud (OPS). Discapacidad. Washington: OPS; [citado 2021 julio 20]. Disponible en: https://www.paho.org/es/temas/discapacidad.

2. Lee BB, Cripps RA, Fitzharris M, Wing PC. The global map for traumatic spinal cord injury epidemiology: update 2011, global incidence rate. Spinal Cord. 2014; 52(2):110-6. Disponible en: https://doi.org/10.1038/sc.2012.158.

3. Cowan LJ, Ahn H, Flores M, Yarrow J, Barks LS, Garvan C, et al. Pressure Ulcer Prevalence by Level of Paralysis in Patients With Spinal Cord Injury in Long-Term Care. Adv Skin Wound Care. 2019;32(3):122-30. Disponible en: https://doi.org/10.1097/01.ASW.0000553109.70752.bf.

4. Bogie KM, Bader DL. Susceptibility of Spinal Cord-Injred Individuals to Pressure Ulcers. En: Bader DL, Bouten C, Colin D, Oomens CWJ, editors. Pressure Ulcer Researh Current and Future Perspective. Berlin: Springer; 2005. p. 73-88.

5. Zakrasek EC, Creasey G, Crew JD. Pressure ulcers in people with spinal cord injury in developing nations. Spinal Cord. 2015; 53(1):7-13. Disponible en: https://doi.org/10.1038/sc.2014.179.

6. Armstrong W, Borg J, Krizack M, Lindsley A, Mines K, Pearlman J, et al. Pautas para el suministro de sillas de ruedas manuales en entornos de menores recursos. Ginebra: Organización Mundial de la Salud; 2008 [citado julio 20 de 2021]. Disponible en: https://www.who.int/disabilities/publications/technology/wheelchairguidelines_sp_finalforwe b.pdf.

7. Borg J. Assistive Technology, Human rights and poverty in developing countries. Perspectives based on a study in Bangladesh [tesis doctoral]. Suecia: Faculty of Medicine, Lund University; 2011.

8. Organización Mundial de la Salud (OMS). ¿Qué calidad de vida?. Foro mundial de la salud. 1996 [citado julio 20 de 2020]; 17(4)385-7. Disponible en: https://apps.who.int/iris/handle/10665/55264.

9. Velarde-Jurado E, Ávila-Figueroa C. Consideraciones metodológicas para evaluar la calidad de vida. Salud Pública Mex. 2002;44(5):448-63.

10. Borg J, Lindström A, Larsson S. Assitive technology in developing countries: national and international responsabilities to implement the Convention on the Rights of Persons with Disabilities. Lancet. 2009;374(9704):1863-5. Disponible en: https://doi.org/10.1016/S0140-6736(09)61872-9.

11. Consortium for Spinal Cord Medicine. Directrices de prácticas clínicas. Úlceras por Decúbito: Lo Que Usted Debe Saber. Washington D.C.: Consortium for Spinal Cord Medicine; 2000.

12. Whiteneck GG, Brooks CA, Charlifue S, Gerhart KA, Mellick D, Overholse D, et al. Craig Handicap Assessment and Reporting Technique. Englewood, Colorado: Craig Hospital; 1992.

13. World Health Organization (WHO). WHOQOL -BREF: Measuring Quality of Life. Ginebra: WHO; 1996.

14. Lyder CH. Medico-Legal Implications. En: Bader DL, Bouten C, Colin D, Oomens CWJ, editors. Pressure Ulcer Researh Current and Future Perspective. Berlin: Springer; 2005. p. 23-34.

15. Basu S, Shukla V. Complications of Wound Healing. En: Mani R, Romanelli M, Shukla V, editors. Measurements in Wound Healing Science and Practice. Berlín: Spriger; 2012. p. 109-144.

Valoración de un programa para la provisión de sillas de ruedas adecuadas para pacientes con lesión medular tomando como indicadores la calidad de vida y la prevención de úlceras por presión 